\title{
Efficient and Documented Preparation of Pharmaceutical Particles for Correlative Microscopy Analyses using mPrep ${ }^{\text {TM }}$ Capsule Processing
}

\author{
Steven L. Goodman
}

Microscopy Innovations LLC, 213 Air Park Rd, Suite 101, Marshfield, WI, 54449, USA.

Pharmaceutics utilize many types of nanometer, micrometer and larger particles that require analyses to determine morphology, uniformity, and chemistry. This, in turn, requires several analytical instruments and multiple specimen preparations. Two additional challenges in the pharma lab are that analyses always seem to be required immediately, and that specimen prep must be documented and reproducible to meet GLP (Good Lab Practice) and similar quality standards. In a prior report we described how documentation and correlative microscopy are valuable for obtaining evidence for intellectual property litigation on tablet formulations [1]. In the present study, an efficient, rapid and GLP-level correlative approach was used to prepare and examine formulations of two types of pharmaceutical particles: siRNA (small interfering RNA) nanoparticles, and sub-millimeter hydrogel drug delivery particles.

Two nanoparticle siRNA drug formulations were prepared for transmission electron microscopy (TEM) to assess morphology and uniformity. Formulations were prepared with and without a positive stain, and with and without uranyl acetate negative staining, for a total of 8 preparations. Four grids of each preparation were simultaneously prepared using $16 \mathrm{mPrep} / \mathrm{g}^{\mathrm{TM}}$ (grid) capsules, an 8-channel $200 \mu \mathrm{l}$ pipettor and a 96-well microtiter plate: First, 2 Formvar-filmed grids were inserted into the $\mathrm{mPrep} / \mathrm{g}$ capsules and then $2 \mathrm{mPrep} / \mathrm{g}$ capsules were stacked onto each pipettor channel to provide 4 grids/channel (Fig 1). The nanoparticle suspensions and other reagents were put into the microtiter plate with each reagent in one well, and each reagent step in a single row. Simultaneous preparation was achieved by first pipetting in $80 \mu \mathrm{l}$ of the nanoparticle suspensions from the first microtiter row into the stacked $\mathrm{mPrep} / \mathrm{g}$ capsules. The nanoparticles were then adsorbed onto the filmed-grids for 30 minutes. The suspensions were then emptied from the pipettor, and the pipetter was moved to the next row for a water rinse, then to the next row for the positive stain (stain in half the microtiter wells and water in the other half) thereby staining half of the grids. After staining, the reagent was emptied from the pipettor and the pipettor was moved sequentially to the remaining rows for a water rinse, negative stain (half the capsules), and water rinse again. The particles were then examined with an FEI Tecnai T-12.

Hydrogel micro-particles were prepared and analyzed to determine chemistry and composition in the dry state from thin cross-sections. The particles were prepared by entrapping each formulation into 8 $\mathrm{mPrep} / \mathrm{s}^{\mathrm{TM}}$ (specimen) capsules (Fig 2a). These mPrep/s capsules were then attached to an 8-channel pipettor and the particles were then simultaneously rinsed in 100\% acetone followed by epoxy infiltration and embedment, in the same manner as shown for reagent delivery in Fig 1a. Epoxy-filled capsules were then ejected (without emptying) into a bottom-sealing $\mathrm{mPrep} / \mathrm{bench}^{\mathrm{TM}}$ microtiter plate, and cured at $60 \mathrm{C}$ (Fig 2b). After curing the mPrep/s capsules were mounted in a microtome chuck and faced thorough the capsule for sectioning (Fig 2c). Dry sections on coverslips were examined with infrared (FTIR) spectroscopy for chemical composition, and sections on SEM stubs were examined for elemental composition with energy dispersive spectroscopy (EDS) (Figs 2d-e). Additional particles were examined with SEM (Fig 2f) and in the hydrated state by light microscopy.

Multichannel template-guided pipetting with mPrep capsules provided rapid, simultaneous preparation 
of 16 TEM grids from 8 nanoparticle preparations, and 8 micro-particle specimen blocks. All were prepared with ease and experimental reproducibility. Since specimens were always labeled throughout the easily followed template-guided protocol, this also provided GLP-level documentation.

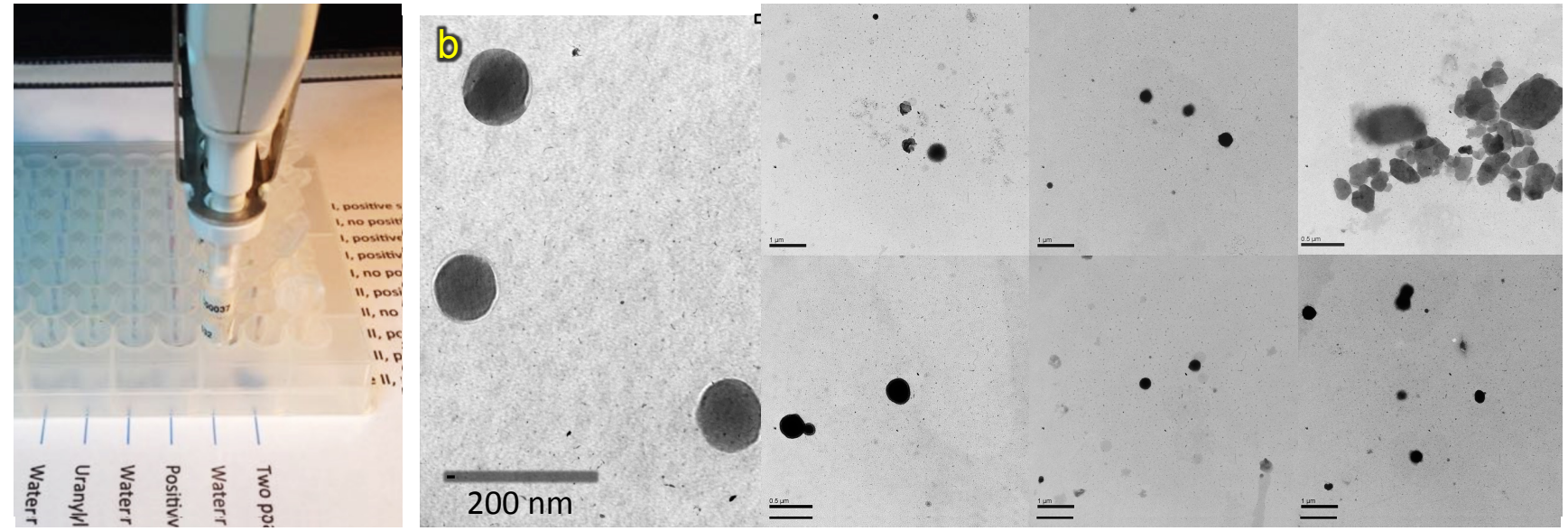

Fig 1: a) Two mPrep/g capsules on each pipettor channel with sample preparation by pipetting reagents from each microtiter row guided by underlying template. b) TEM of siRNA nanoparticle preparations.
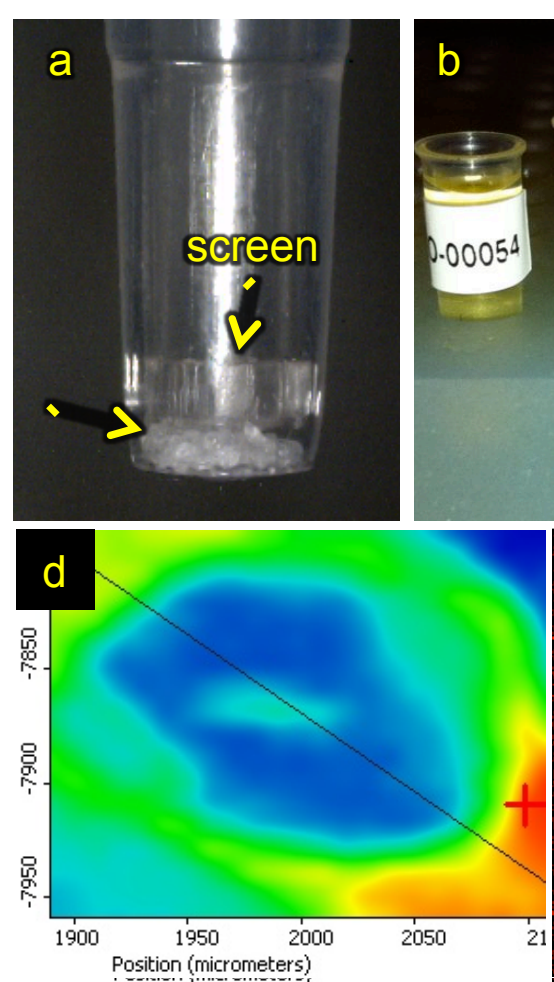
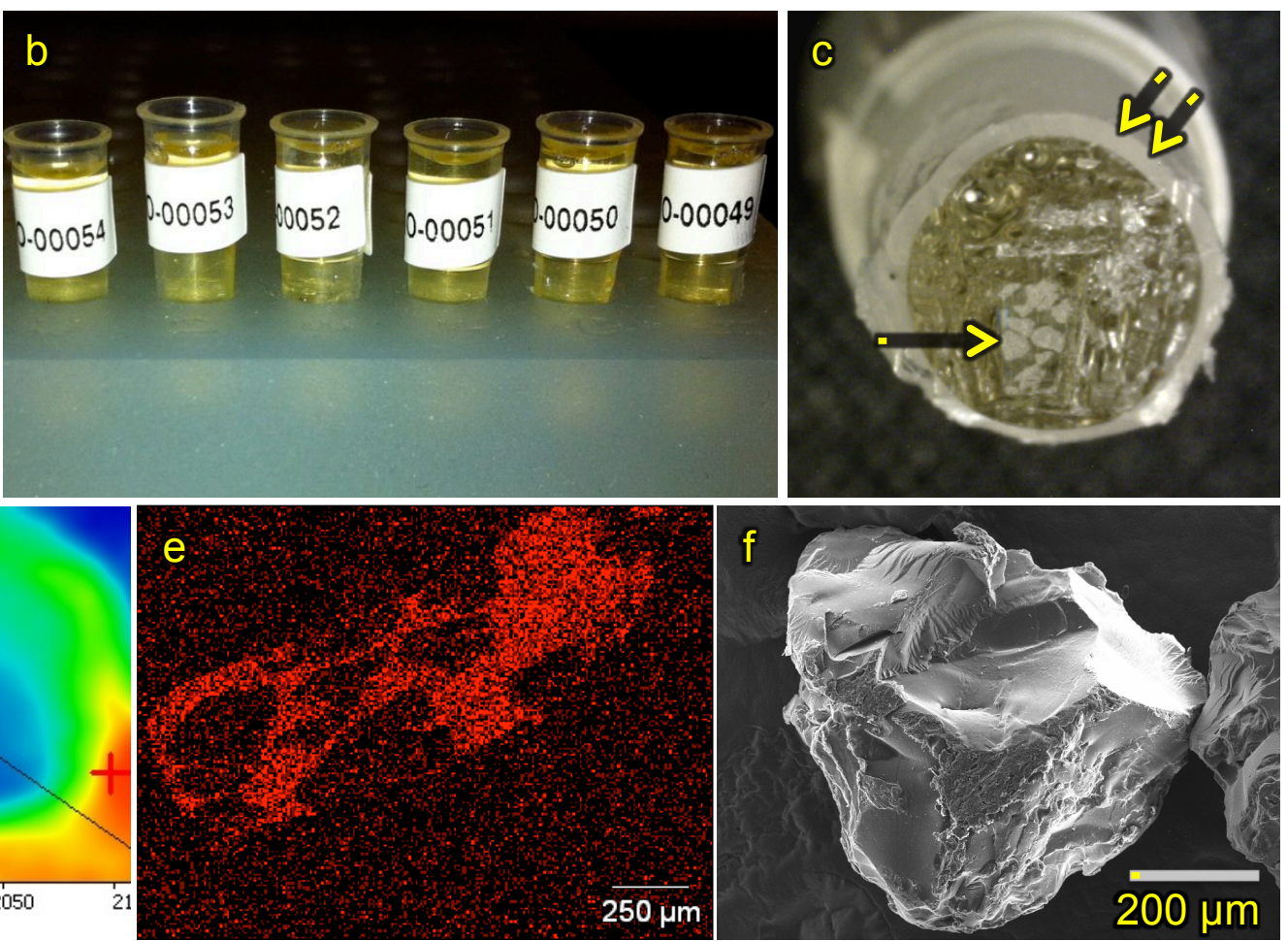

Fig 2: a) Dry hydrogel micro-particles entrapped in mPrep/s capsule (arrow) by removable adjustable screen. b) mPrep/s blocks containing particles after epoxy curing in bottom sealing mPrep/bench microtiter plate. c) mPrep/s capsule with embedded particles in microtome chuck. Several visible crosssectioned particles (arrow). Note capsule trimmed away for sectioning (double arrow). FTIR (d) and SEM-EDS maps (e) from particle cross-sections. f) Whole particle imaged with FE-SEM.

[1] SL Goodman, JJ Edwards, Microsc Microanal, 20 (suppl 3) (2014) 1416-7. 